DOI: http://dx.doi.org/10.12957/demetra.2015.14451

\title{
Estado nutricional e indicadores de qualidade em terapia nutricional enteral em pacientes institucionalizados com paralisia cerebral
}

\section{Nutritional status and quality indicators for enteral nutritional therapy in institutionalized patients with cerebral palsy}

Eduardo Rodrigo de Lara Luz'

Thais Regina Mezzomo²

' Centro Universitário Campos de Andrade,
Centro de Ciências da Saúde, Curso de Nutrição.
Curitiba-PR, Brasil.

Correspondência / Corrrespondence

Thais Regina Mezzomo

e-mail: thaismezzomo@yahoo.com.br

\section{Resumo}

O objetivo do estudo foi descrever o estado nutricional (EN) de pacientes com paralisia cerebral institucionalizados com terapia nutricional enteral (TNE) e aplicar indicadores de qualidade referentes à administração de nutrição enteral (NE). Estudo retrospectivo observacional. Para avaliar o EN, foram coletados nos prontuários dos indivíduos dados referentes a peso corporal, estatura, circunferência do braço (CB), prega cutânea tricipital (PCT), circunferência muscular do baço $(\mathrm{CMB})$ e índice de massa corporal (IMC). Foram monitorados seis indicadores de qualidade: (1) frequência de medida de IMC em pacientes com terapia nutricional (TN); (2) frequência de medida ou estimativa de gasto energético e necessidade proteica em pacientes em TN; (3) frequência de dias de administração adequada de líquidos em pacientes em TN; (4) frequência de dias de administração adequada de energia em pacientes em TN; (5) frequência de dias de administração adequada de proteína em pacientes em $\mathrm{TNe}$ (6) frequência de intercorrências relacionadas à administração de NE.Foram avaliados oito indivíduos. A média de IMC foi 18,65 $\pm 3,2$ $\mathrm{kg} / \mathrm{m}^{2}$; o \% CB foi de 85,63 $\pm 6,05 \%$; o \% CMB foi de 85, $13 \pm 15,27 \%$; e o \%PCT foi de 116,5 \pm 76,6\%. Com relação aos indicadores 4 e 6 , foram percebidos $25 \%$ de adequação, e o indicador 5 apresentou $75 \%$ de adequação. Os demais indicadores apresentaram 100\% de conformidade. Os pacientes apresentaram depleção de tecido muscular e reserva de tecido adiposo, apesar do baixo-peso. Os indicadores 4, 5 e 6 apresentaram baixa porcentagem de adequação, refletindo a importância de seu monitoramento.

Palavras-chave: Paralisia Cerebral. Estado Nutricional. Indicadores de Qualidade. Nutrição Enteral. 


\section{Abstract}

This study aimed to describe the nutritional status (NS) of institutionalized patients with cerebral palsy with enteral nutrition therapy (ENT) and apply quality indicators concerning administration of enteral nutrition (EN). Retrospective observational study. To assess NS, data on body weight, height, arm circumference (AC), triceps skinfold thickness (TSF), muscle circumference spleen (MCS) and body mass index (BMI) were collected from medical records of individuals. Six quality indicators were monitored: (1) frequency of BMI measurement in patients with nutritional therapy (NT); (2) frequency measurement or estimation of energy expenditure and protein needs of patients in NT; (3) frequency of days of adequate fluid administration in patients in NT; (4) frequency of days of proper management of energy in patients in NT; (5) frequency of days of proper administration of protein in patients with NT; and (6) frequency of complications related to the administration of EN. Eight individuals were assessed. The mean BMI was $18.65 \pm 3.2$ $\mathrm{kg} / \mathrm{m}^{2}$; \%AC was $85.63 \% \pm 6.05 \%$; MCS was $85.13 \% \pm 15.27 \%$; and TSF was $116.5 \% \pm 76.6 \%$. Regarding indicators 4 and 6 , it was perceived $25 \%$ of adequacy, and 5 showed $75 \%$ of adequacy. The other indicators showed $100 \%$ compliance. The patients presented depletion of muscle tissue and fat reserve, despite low weight. Indicators 4,5 and 6 presented low percentage of adequacy, reflecting the importance of their monitoring.

Key words: Cerebral Palsy. Nutritional Status. Quality Indicators. Enteral Nutrition.

\section{Introdução}

A paralisia cerebral (PC) é atribuída a um grupo de desordens permanentes no desenvolvimento do movimento e da postura, gerando limitações nas atividades. Essas desordens são atribuídas a distúrbios não progressivos que ocorrem durante o desenvolvimento cerebral fetal ou na infância. ${ }^{1}$

A PC possui efeitos importantes na funcionalidade e na qualidade de vida de indivíduos e seus familiares. ${ }^{2}$ As desordens motoras da PC são frequentemente acompanhadas de distúrbios de sensação, cognição, comunicação, comportamento e com presença de convulsões. ${ }^{1}$ 
A PC é a mais frequente causa de incapacidade na infância, acometendo 2-2,8/1.000 nascidos vivos, ${ }^{3}$ podendo chegar a sete crianças para cada mil nascidos vivos em países subdesenvolvidos. ${ }^{4}$ Sugere-se que os elevados números encontrados em países subdesenvolvidos sejam devidos aos cuidados precários fornecidos às gestantes e aos recém-nascidos em muitas regiões. ${ }^{2}$

Os indivíduos com PC podem apresentar distúrbios gastrointestinais e dificuldades para alimentação, que repercutem diretamente em seu estado nutricional e qualidade de vida, visto que a conexão neurológica com o trato digestório é de primordial importância. ${ }^{5,6}$ Dentre as dificuldades mais encontradas, encontram-se a disfagia para sólidos e líquidos, as regurgitações, os vômitos, o tempo prolongado para ofertar a refeição e a constipação intestinal. ${ }^{6-8}$

A terapia nutricional enteral (TNE) surge como uma possibilidade terapêutica de manutenção ou recuperação do estado nutricional para os indivíduos que apresentam o trato gastrintestinal íntegro, mas com a ingestão oral parcial ou totalmente comprometida. A nutrição enteral (NE), portanto, visa oferecer todos os nutrientes necessários para a manutenção da vida, o crescimento celular e tecidual, minimizando e/ou revertendo o impacto da desnutrição. ${ }^{9}$

A TNE precoce pode ser um importante fator na promoção da saúde, diminuição do estresse fisiológico e manutenção da imunidade. ${ }^{10}$ Nesse cenário, a escolha e a prescrição da NE é complexa e implica conhecimento clínico e nutricional. Além disso, tão importante quanto a prescrição da TNE adequada às necessidades do paciente, é a certeza de que o paciente efetivamente receberá o volume prescrito. ${ }^{8} \mathrm{O}$ aporte insuficiente de nutrientes torna-se ainda mais crítico em presença de desnutrição. ${ }^{11-13}$

A adequação da oferta energética ao paciente com PC é necessária para o reestabelecimento do estado nutricional. Entretanto, frequentemente ocorre uma suboferta frente às estimativas nutricionais. São vários os fatores que interferem na oferta real, dentre eles, a intolerância à dieta e a interrupção da administração da via nutricional para procedimentos. ${ }^{14}$

A monitoração diária da oferta nutricional, nesse contexto, é essencial, pois permite que estratégias sejam estabelecidas para aumentar a eficiência da terapia nutricional e, consequentemente, melhorar a qualidade na assistência nutricional. ${ }^{15}$ Nesse contexto, os indicadores de qualidade são aplicados nas instituições, a fim de garantir a eficiência nas rotinas diárias, redução de custos, maior capacidade de análise de processos e principalmente, melhores resultados clínicos e de qualidade de vida para o indivíduo.

Dessa forma, foi objetivo deste estudo descrever o estado nutricional de pacientes com PC institucionalizados e com TNE e aplicar indicadores de qualidade referentes à administração de nutrição enteral. 


\section{Metodologia}

Foi realizado estudo retrospectivo observacional com pacientes adultos em nutrição enteral com diagnóstico de PC, institucionalizados em uma instituição pública no município de Curitiba-PR. O presente estudo foi aprovado pelo Comitê de Ética em Pesquisa do Centro Universitário Campos de Andrade sob no 817.867. A coleta de dados ocorreu em setembro de 2014, após a assinatura do Termo de Consentimento Livre e Esclarecido, pelo responsável pela instituição.

Foram incluídos na pesquisa todos os pacientes que recebiam TNE exclusiva por no mínimo 72 horas, com idade mínima de 20 anos, de ambos os sexos. Para a identificação dos pacientes, foram obtidas as seguintes informações no prontuário médico: nome, sexo e data de nascimento. Os dados clínicos coletados foram: diagnóstico médico, presença de comorbidades e exames bioquímicos de hemoglobina, albumina, colesterol total, triglicérides e glicemia. Os valores dos exames bioquímicos foram comparados com Verrastro. ${ }^{16}$

\section{Avaliação do estado nutricional}

Para avaliar o estado nutricional, foram coletados nos prontuários dos pacientes avaliados o peso atual ou estimado, a estatura atual ou estimada, a circunferência do braço (CB), a prega cutânea tricipital (PCT), a circunferência muscular do braço (CMB) e o índice de massa corporal (IMC). A classificação do estado nutricional da CB, PCT e CMB foi realizada de acordo com Blackburn \& Thornton. ${ }^{17}$ A classificação do IMC foi classificada de acordo com $\mathrm{WHO}^{18}$ para adultos (20 a 59 anos).

\section{Indicadores de qualidade em terapia nutricional}

\section{Comparação da dieta programada e administrada em pacientes com terapia nutricional}

Foi avaliada a adequação entre a dieta enteral programada e administrada perante calorias, proteínas carboidratos, fibras, consumo hídrico e a adequação do valor energético total (VET).

Frequência de medida de IMC em pacientes com terapia nutricional

Este indicador foi analisado considerando o número de pacientes em TN com o valor calculado do IMC, dividido pelo número total de pacientes em TN, multiplicado por 100. As informações foram coletadas nos prontuários dos pacientes. A meta para o indicador foi estipulada em $80 \% .^{19}$ 
Frequência de medida ou estimativa de gasto energético e necessidade proteica em pacientes em terapia nutricional

Este indicador foi mensurado de acordo com o número de pacientes em TN que fizeram avaliação dos gastos energético e proteico, dividido pelo número total de pacientes em TN, multiplicado por 100. As informações foram coletadas nos prontuários dos pacientes. A meta para o indicador foi estipulada em $80 \% .^{19}$

Frequência de dias de administração adequada de energia em pacientes em terapia nutricional

Este indicador foi calculado de acordo com o número de dias com aporte calórico menor que $25 \mathrm{kcal} / \mathrm{kg}$ ou maior que $35 \mathrm{kcal} / \mathrm{kg} / \mathrm{dia}$, dividido pelo número total de dias no período avaliado, em seguida, multiplicado por 100. As informações foram coletadas nos prontuários dos pacientes. A meta para o indicador foi estipulada em $80 \% .^{19}$

Frequência de dias de administração adequada de proteína em pacientes em terapia nutricional

Esse indicador foi analisado considerando o número de dias com aporte proteico menor que $0,8 \mathrm{~g} / \mathrm{kg} / \mathrm{dia}$ ou maior que $2 \mathrm{~g} / \mathrm{kg} / \mathrm{dia}$, dividido pelo número total de dias no período avaliado, em seguida, multiplicado por 100. As informações foram coletadas nos prontuários dos pacientes. A meta para o indicador foi estipulada em $80 \% .^{19}$

Frequência de dias de administração adequada de líquidos em pacientes em terapia nutricional

Este indicador foi calculado de acordo com o número de dias com aporte líquido inferior a $1 \mathrm{kcal} / \mathrm{ml}$, dividido pelo número total de dias no período avaliado, em seguida, multiplicado por 100. As informações foram coletadas nos prontuários dos pacientes. A meta para o indicador foi estipulada em $90 \% .^{19}$

Frequência de intercorrências relacionadas à administração de nutrição enteral

Este indicador foi estipulado de acordo com o número de pacientes que apresentaram intercorrências como náuseas, vômitos, estase gástrica, refluxo, cólica, distensão abdominal, flatulência, constipação intestinal e outras intercorrências relevantes para TNE, dividido pelo número total de pacientes em NE, multiplicado por 100. As informações foram coletadas nos prontuários dos pacientes. A meta para o indicador foi estipulada em $5 \% .{ }^{19}$ 


\section{Análise estatística}

Os dados foram descritos por meio de distribuição de frequência simples, média e desvio padrão e compilados no programa Microsoft Excel®.

\section{Resultados}

Dos 209 pacientes institucionalizados, foram incluídos no estudo oito pacientes que contemplaram os critérios de inclusão, sendo $62,5 \%(\mathrm{n}=5)$ do sexo feminino e $37,5 \%$ $(\mathrm{n}=3)$ do sexo masculino, com média de idade de 29,5 \pm 9,62 anos. O diagnóstico médico desses pacientes encontra-se na figura 1. A maioria dos pacientes, $67,5 \%(n=5)$, apresenta diagnóstico de paralisia cerebral diplégica e 37,5\% (n=3) apresentam paralisia cerebral quadriplégica. Dentro da classificação de PC, $50 \%(\mathrm{n}=4)$ apresentam deficiência intelectual; 37,5\% $(\mathrm{n}=3)$ apresentam microcefalia; $25 \%(\mathrm{n}=2)$ apresentam deficiência visual; e $25 \%(\mathrm{n}=2)$ apresentam epilepsia.

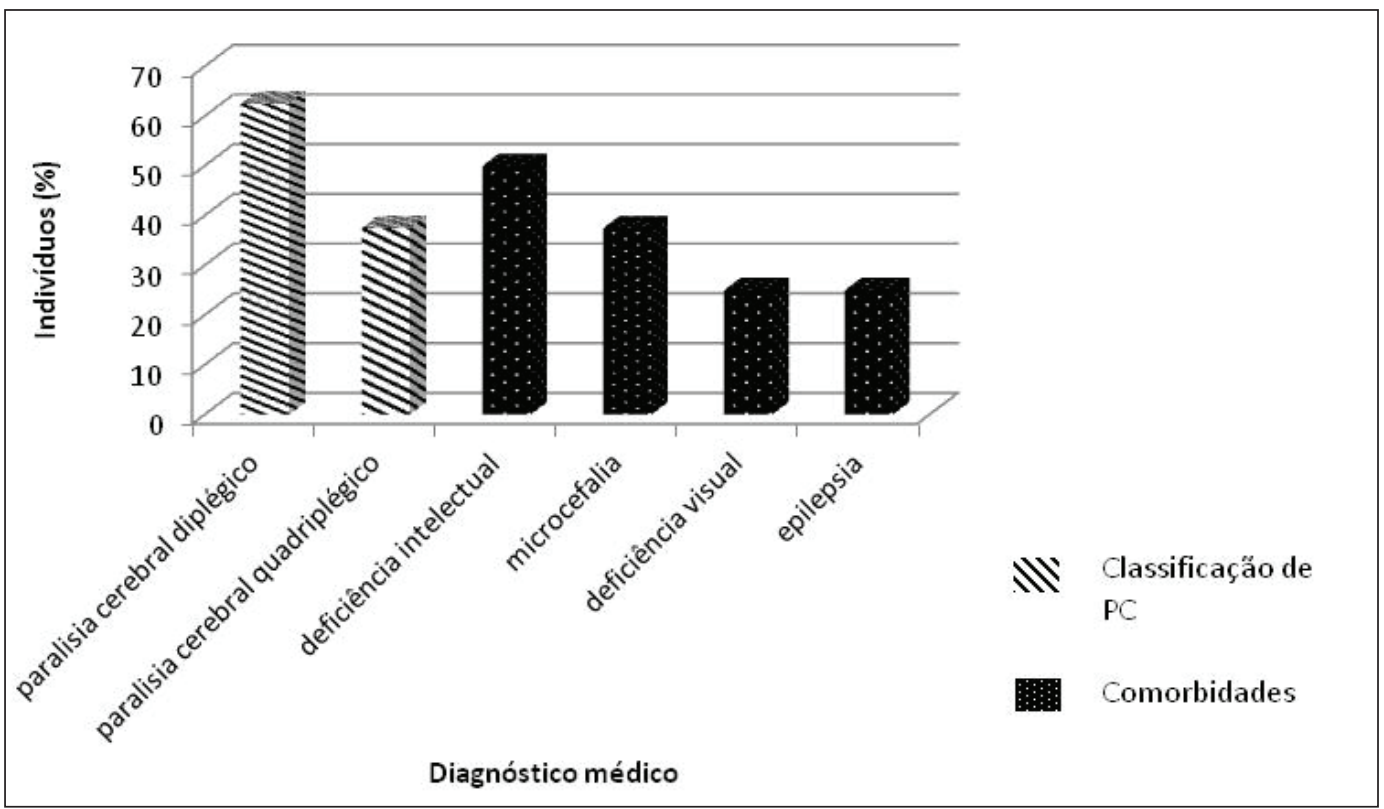

Legenda: PC: Paralisia Cerebral.

Figura 1. Diagnóstico médico dos indivíduos com paralisia cerebral em nutrição enteral avaliados no estudo, Curitiba-PR, 2014. 
A descrição do estado nutricional está ilustrada na tabela 1. De acordo com esses resultados, pode-se observar que $62,5 \%(n=5)$ dos indivíduos encontraram-se com IMC de magreza grau I e 37,5\% $(n=3)$ estão eutróficos. Em relação à \%CB, 12,5\% (n= 1) apresentam desnutrição moderada; $62,5 \%(n=5)$, desnutrição leve; e $25 \%(n=2)$ estão eutróficos. Já em relação ao \%PCT, 12,5\% (n=1) apresentam desnutrição grave; 12,5\% $(n=1)$, desnutrição moderada; $12,5 \%(n=1)$, desnutrição leve; 37,5\% (n= 3), eutrofia; e $25 \%(\mathrm{n}=2)$, obesidade. No que se refere à \%CMB, $50 \%(\mathrm{n}=4)$ apresentam desnutrição leve; $25 \%(n=2)$, desnutrição grave; e $25 \%(n=2)$ estão eutróficos.

Tabela 1. Valores médios e desvio padrão de parâmetros de avaliação do estado nutricional dos indivíduos com paralisia cerebral avaliados no estudo, Curitiba-PR, 2014.

\begin{tabular}{|c|c|c|c|}
\hline & & Média & DP \\
\hline \multirow[t]{3}{*}{ IMC $\left(\mathrm{kg} / \mathrm{m}^{2}\right)$} & & 18,65 & 3,29 \\
\hline & Magreza grau I & $62,5 \%$ & $(n=5)$ \\
\hline & Eutrofia & $37,5 \%$ & $(\mathrm{n}=3)$ \\
\hline \multirow[t]{4}{*}{$\mathrm{CB}(\%)$} & & $85,6 \%$ & 6,05 \\
\hline & Desnutrição moderada & $12,5 \%$ & $(\mathrm{n}=1)$ \\
\hline & Desnutrição leve & $62,5 \%$ & $(\mathrm{n}=5)$ \\
\hline & Eutrófico & $25 \%$ & $(\mathrm{n}=2)$ \\
\hline \multirow[t]{6}{*}{$\operatorname{PCT}(\%)$} & & $116,5 \%$ & 76,6 \\
\hline & Desnutrição grave & $12,5 \%$ & $(\mathrm{n}=1)$ \\
\hline & Desnutrição moderada & $12,5 \%$ & $(\mathrm{n}=1)$ \\
\hline & Desnutrição leve & $12,5 \%$ & $(n=1)$ \\
\hline & Eutrófico & $37,5 \%$ & $(\mathrm{n}=3)$ \\
\hline & Obesidade & $25 \%$ & $(n=2)$ \\
\hline \multirow[t]{4}{*}{ CMB $(\%)$} & & $85,13 \%$ & 15,27 \\
\hline & Desnutrição grave & $25 \%$ & $(\mathrm{n}=2)$ \\
\hline & Desnutrição leve & $50 \%$ & $(n=4)$ \\
\hline & Eutrófico & $25 \%$ & $(n=2)$ \\
\hline
\end{tabular}

Legenda: IMC: Índice de massa corporal; CB: circunferência do braço; PCT: prega cutânea tricipital; CMB: circunferência muscular do braço. 
Os dados apresentados na tabela 2 apresentam as médias dos exames laboratoriais dos indivíduos. Os exames demonstram que 37,5\% (n=3) dos indivíduos apresentam anemia, caracterizada pelos baixos níveis de hemoglobina, sendo $25 \%(2=2)$ do sexo masculino e 12,5\% $(n=1)$ do sexo feminino. Com relação à albumina, apenas 12,5\% $(n=1)$ apresentam hipoalbuminemia. No que se refere ao colesterol, 12,5\% (n=1) apresentam hipercolesterolemia. Os exames de triglicérides e glicemia apresentaram-se dentro dos valores de normalidade.

Tabela 2. Exames laboratoriais dos indivíduos com paralisia cerebral avaliados no estudo, Curitiba, PR, 2014.

\begin{tabular}{cc}
\hline Exame laboratorial & Média \pm DP \\
\hline Hemoglobina $(\mathrm{g} / \mathrm{dl})$ & $12,98 \pm 1,23$ \\
Albumina $(\mathrm{g} / \mathrm{dl})$ & $4,06 \pm 0,47$ \\
Colesterol $(\mathrm{mg} / \mathrm{dl})$ & $157,78 \pm 33,57$ \\
Triglicérides $(\mathrm{mg} / \mathrm{dl})$ & $79,73 \pm 30,07$ \\
Glicemia $(\mathrm{mg} / \mathrm{dl})$ & $80,51 \pm 13,95$ \\
\hline
\end{tabular}

Em relação à alimentação enteral, 100\% dos pacientes que estavam sob terapia nutricional enteral tinham como via de acesso a gastrostomia. As dietas enterais administradas eram do tipo polimérica em 87,5\% $(\mathrm{n}=7)$ dos indivíduos. Apenas $12,5 \%(\mathrm{n}=1)$ utilizavam dieta enteral hidrolisada. A tabela 3 compara os valores programados para dieta enteral, bem como os valores administrados e o percentual de adequação da dieta.

Tabela 3. Comparação entre dieta programada e administrada em indivíduos com paralisia cerebral avaliados no estudo, Curitiba-PR, 2014.

\begin{tabular}{cccc}
\hline & Programado & Administrado & \% adequação \\
\hline VET & $1493,63 \pm 110,25$ & $1490,5 \pm 551,74$ & $100,35 \pm 37,52$ \\
kcal/kg/dia & $34,6 \pm 4,32$ & $35,15 \pm 15,95$ & $100,21 \pm 37,32$ \\
Proteína (g/kg/dia) & $1,12 \pm 0,06$ & $1,25 \pm 0,44$ & $111,97 \pm 39,75$ \\
Carboidrato (\% VET) & $55,36 \pm 7,49$ & $60 \pm 0$ & $92,26 \pm 12,49$ \\
Lipídeos (\% VET) & $29,94 \pm 2,11$ & $29,44 \pm 6,15$ & $110,6 \pm 27,07$ \\
Fibras (g/dia) & $20 \pm 0$ & $14,89 \pm 3,22$ & $74,46 \pm 16,09$ \\
Consumo hídrico (kcal/ml) & $1,48 \pm 0,10$ & $1,84 \pm 0,38$ & $124,54 \pm 24,28$ \\
\hline
\end{tabular}

*Resultados expressos em valores médios e desvio-padrão. 
Em relação à alimentação enteral, não houve grande diferença do VET programado e o administrado. As adequações de valor energético total, $\mathrm{kcal} / \mathrm{kg}$ de peso ao dia, proteínas, carboidratos e lipídios apresentaram-se entre 92,26 a 110,6\%. O consumo de fibras apresentou-se inferior ao recomendado em 74,46\% $\pm 16,09 \%$, e o consumo hídrico apresentou-se superior $(124,54 \pm 24,28 \%)$.

Ao mensurar os indicadores de frequência da medida de IMC nos pacientes em TNE, de frequência de medida ou estimativa de gasto energético e necessidade proteica em pacientes em terapia nutricional e frequência de dias de administração adequada de líquidos em pacientes em terapia nutricional, observou-se que $100 \%$ deles apresentam conformidade nesses indicadores.

Com relação ao indicador de frequência de dias de administração adequada de energia em pacientes em TNE, observou-se que 37,5\% $(\mathrm{n}=3)$ dos indivíduos recebem menos que $25 \mathrm{kcal} / \mathrm{kg} / \mathrm{dia}$ e $37,5 \%(\mathrm{n}=3)$ dos pacientes recebem mais do que $35 \mathrm{kcal} / \mathrm{kg} / \mathrm{dia}$ dessa forma este indicador apresentou $25 \%$ de adequação. Com relação ao indicador de frequência de administração adequada de proteínas, observou-se que 12,5\% (n=1) recebem menos que $0,8 \mathrm{~g} / \mathrm{kg} / \mathrm{dia}$ de proteínas e $12,5 \%(\mathrm{n}=1)$ dos pacientes recebem mais do que $2 \mathrm{~g} / \mathrm{kg} / \mathrm{dia}$ - ou seja, este indicador apresentou $75 \%$ de adequação.

A figura 2 ilustra as intercorrências relacionadas à administração de NE nos pacientes avaliados. Com relação a este indicador, observou-se que 75\% $(n=6)$ dos pacientes apresentaram alguma intercorrência, sendo a principal a constipação em $75 \%$ (n= 6) dos indivíduos, seguida por náuseas e vômitos com 12,5\% $(n=1)$. Nenhuma outra intercorrência foi apresentada pelos pacientes no período avaliado.

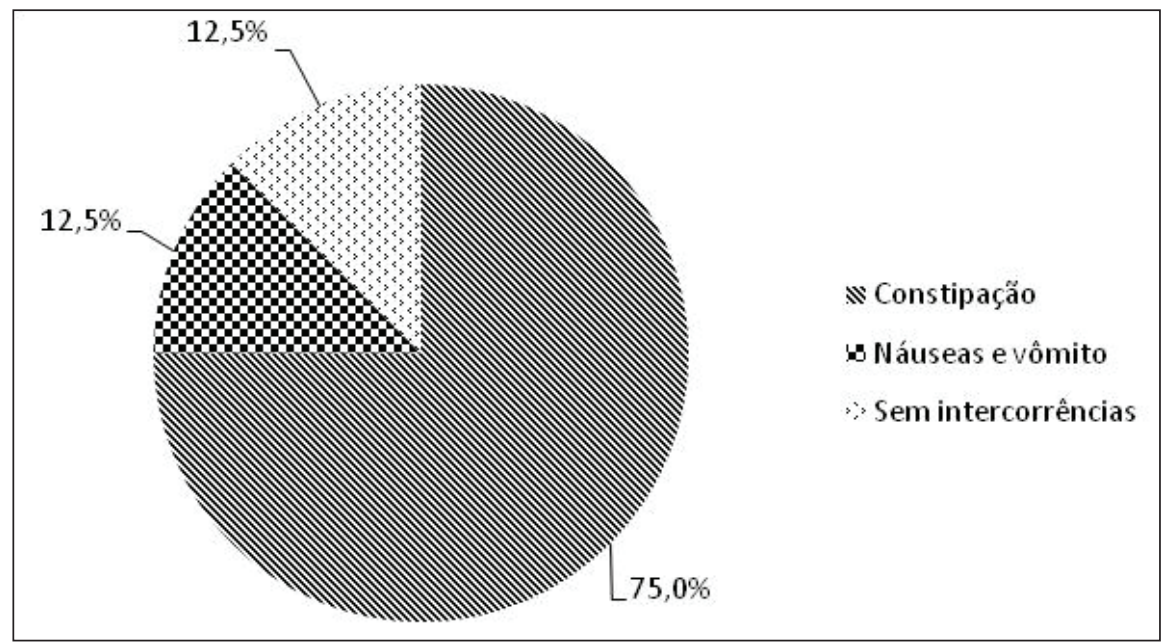

Figura 2. Intercorrências relacionadas à administração de dieta enteral apresentadas pelos pacientes com paralisia cerebral avaliados no estudo, Curitiba-PR, 2014. 


\section{Discussão}

A TNE tem sido considerada, sempre que possível, como o método de escolha para pacientes que não possuem condições de alimentação por via oral suficientes para atingir o mínimo de suas necessidades energéticas, normalmente aumentadas pelo estresse fisiológico. ${ }^{18}$

No presente estudo, pode-se observar que a maioria dos indivíduos avaliados apresenta magreza grau I, segundo o IMC. Mesmo quando adequadamente nutridos, indivíduos com PC são menores que pessoas que não têm deficiência, possivelmente em razão de inatividade física, forças mecânicas sobre ossos, articulações e musculatura, fatores endócrinos, altas prevalências de prematuridade e baixo-peso ao nascer. ${ }^{20,21}$ Poucos estudos avaliam o estado nutricional de indivíduos com paralisia cerebral em adultos. Os estudos encontrados sugerem que há diminuição do crescimento no linear, no ganho de peso e alterações na composição corporal, como decréscimo na massa muscular, massa gorda e densidade óssea. Linnea et al. ${ }^{22}$ relacionam o estado nutricional inadequado como possível consequência de ingestão calórica insuficiente. Contudo, segundo o Ministério da Saúde, atingir índices antropométricos de peso e altura definidos em populações gerais não deve constituir meta ideal quando se trata de saúde e crescimento de indivíduos com PC. ${ }^{23}$ Até o momento, não há valores de IMC específicos para indivíduos adultos com PC.

De acordo com a avaliação e adequação da PCT, pode-se observar que os pacientes apresentaram sobrepeso, o que indica reservas de gordura corporal, ${ }^{24}$ entretanto, não foram avaliados outros compartimentos corporais, ${ }^{25}$ dessa forma esse método pode não ser um bom indicador de EN em indivíduos com PC. No que se refere a CB e CMB, ambos os parâmetros refletem o compartimento de massa magra, e os pacientes apresentaram baixa massa magra e baixo-peso corporal, conforme característica dessa população, segundo estudos. ${ }^{26}$

Os dados bioquímicos dos indivíduos deste estudo estavam dentro dos valores de referência de normalidade, o que pode estar relacionado ao longo tempo do diagnóstico clínico, adaptação do organismo, ou à adequação da TNE. ${ }^{27}$ A albumina sérica é bastante utilizada para verificação de prognóstico, mortalidade e prediz possíveis complicações. ${ }^{28,29}$ A hipocolesterolemia (abaixo de $150 \mathrm{mg} / \mathrm{dl}$ ) tem sido estudada como índice prognóstico em desnutrição, com detecção de aumento da mortalidade e tempo de permanência hospitalar, ${ }^{30}$ enquanto que a hipercolesterolemia é considerada fator de risco para a doença arterial coronariana. ${ }^{31}$ No tocante à concentração de hemoglobina, estudos revelam que o estado nutricional inadequado representa fator de risco para o desenvolvimento da anemia. ${ }^{32,33}$

Conforme observado neste estudo, houve boa tolerância na administração da dieta enteral para esses pacientes, provavelmente por serem pacientes crônicos em nutrição 
enteral, pela atenção disponibilizada aos pacientes pela equipe multidisciplinar, bem como a educação continuada da equipe. ${ }^{34,35}$

Os indicadores de qualidade em terapia nutricional são úteis para monitorar a prática de TN, pois permitem conhecer sua segurança, eficiência e custo-benefício, além de permitirem, se necessário for, o planejamento de ações corretivas. ${ }^{19,36}$ Os indicadores de frequência da medida de IMC, frequência de medida ou estimativa de gasto energético e necessidade proteica em pacientes em terapia nutricional apresentaram 100\% de aprovação, pois fazem parte dos protocolos padrões da instituição.

Com relação aos dias de administração adequada de energia em pacientes em terapia nutricional, pode-se perceber que houve $50 \%(\mathrm{n}=4)$ de indivíduos recebendo inadequação calórica, ou seja, com oferta calórica abaixo de $25 \mathrm{kcal} / \mathrm{kg} / \mathrm{dia}$ ou acima de $35 \mathrm{kcal} / \mathrm{kg} / \mathrm{dia}$. Provavelmente, isto está correlacionado com uma necessidade energética subestimada.

Quanto ao indicador de frequência de dias de administração adequada de proteína em pacientes em terapia nutricional, pode-se observar que $12,5 \%(n=1)$ dos pacientes recebem uma oferta de proteína menor que $0,8 \mathrm{~g} / \mathrm{kg} / \mathrm{dia}$ e $12,5 \%$ (n=1) maior que $2,0 \mathrm{~g} / \mathrm{kg} /$ dia, o que pode estar relacionado a uma má distribuição dos macronutrientes da dieta.

Sobre o indicador de frequência de dias de administração adequada de líquidos em pacientes em terapia nutricional, houve $100 \%(\mathrm{n}=8)$ de adequação desse indicador, o que pode estar correlacionado com uma estimativa adequada às necessidades dos pacientes, além da boa aceitação da dieta enteral recebida.

A obstipação intestinal pode ser caracterizada como a ocorrência de menos de uma evacuação no período de três dias. ${ }^{37}$ Nesta pesquisa, a principal intercorrência relatada foi a constipação, em 75\% dos indivíduos avaliados, e sua ocorrência pode afetar a evolução clínica de pacientes submetidos a TNE, por se associar com distensão abdominal, vômitos, obstrução intestinal e perfuração. ${ }^{38}$ A obstipação é uma característica da PC, ${ }^{8}$ e outras causas podem estar relacionadas aos medicamentos associados, consumo de fibras na dieta enteral e desidratação. ${ }^{39,40}$

Entre os medicamentos que retardam a motilidade do trato gastrointestinal, destacamse as classes medicamentosas dos benzodiazepínicos e os opióides. ${ }^{38,41,42}$ A utilização de NE com fibras pode ser considerada um fator de proteção para evitar a obstipação, mas ainda faltam evidências sobre a quantidade e a qualidade da fibra que deve ser indicada para prevenção de distúrbios no trânsito intestinal em pacientes sob TNE exclusiva. ${ }^{43}$ Neste estudo, evidenciou-se que as dietas enterais não atingiram a recomendação preconizada de fibras, o que contribui com a obstipação. 


\section{Conclusão}

A desnutrição apresentou alta prevalência nos pacientes institucionalizados estudados, em que a TNE nesses casos foi a única via de alimentação e nutrição.

A adoção de mecanismos de vigilância clínica, com abordagem de equipe multidisciplinar, criação e utilização de protocolos, indicadores de qualidade e formação continuada dos profissionais de saúde, pode ser importante medida para assegurar a administração adequada da TNE e proporcionar maior benefício aos pacientes. Sendo assim, esta proposta de indicadores tem importância fundamental, considerando as dificuldades existentes na avaliação nutricional do paciente adulto com PC.

\section{Referências}

1. Bax M, Goldstein M, Rosenbaum P, Leviton A, Paneth N, Dan B, et al. Proposed definition and classification of cerebral palsy, April 2005. Dev. Med. Child Neurol. 2005; 47(8):571-576.

2. Mancini MC, Fiúza PM, Rebelo JM, Magalhães LC, Coelho ZA, Paixao ML, et al. Comparison of functional activity performance in normally developing children and children with cerebral palsy. Arq. Neuropsiquiatr. 2002; 60(2-B):446-452.

3. Gladstone MA. Review of the incidence and prevalence, types and aetiology of childhood cerebral palsy in resource-poor settings. Ann. Trop. Paediatr. 2010; 30(3):181-196.

4. Caram ALA, et al. Morcillo AM, Costa Pinto EAL. Estado nutricional de crianças com paralisia cerebral. Rev. Nutr. 2010; 23(2):211-219.

5. Del Giudice E, Staiano A, Capano G, Romano A, Florimonte L, Miele E, et al. Gastrointestinal manifestations in children with cerebral palsy. Brain Dev. 1999; 21(5):307-311.

6. Stevenson RD. Beyond growth: gastrostomy feeding in children with cerebral palsy. Dev. Med. Child Neurol. 2005; 47(2):76.

7. Sullivan PB, Lambert B, Rose M, Ford-Adams M, Johnson A, Griffiths P. Prevalence and severity of feeding and nutritional problems in children with neurological impairment: oxford feeding study. Dev. Med. Child. Neurol. 2000; 42(10):674-680.

8. Callis EA, Veugelers R, Sheppard JJ, Tibboel D, Evenhuis HM, Penning C. Dysphagia in children with severe generalized cerebral palsy and intellectual disability. Dev. Med. Child. Neurol. 2008; 50(8):625-630.

9. Brasil. Ministério da Saúde. Agencia Nacional de Vigilância Sanitária - ANVISA. Resolução RCD no 63, de 6 de julho de 2000. Aprova o Regulamento Técnico para fixar os requisitos mínimos exigidos para a Terapia de Nutrição Enteral. Disponível em: http://www.anvisa.gov.br/legis/ resol/200/63_00rcd. htm.

10. Teixeira ACC, Caruso L, Soriano FG. Terapia nutricional enteral em unidade de terapia intensiva: infusão versus necessidades. Rev. Bras. Ter. Int. 2006; 18(4)331-337. 
11. Campanella LCA, et al. Terapia nutricional enteral: a dieta prescrita e realmente infundida? Rev. Bras. Nutr. Clin. 2008; 23(1):21-25.

12. World Health Organization. Physical status: the use and interpretation of anthropometry. Geneve: WHO; 1995. Technical Report Series, 854.

13. Kurihayashi AY, Caruso L, Soriano FG. Terapia nutricional parenteral em UTI: aplicação dos indicadores de qualidade. O Mundo da Saúde 2009; 33(4):480-487.

14. Elpern EH, Stutz L, Peterson S, Gurka DP, Skipper A. Outcomes associated with enteral tube feedings in a medical intensive Care Unit. Am. J. Crit. Care 2004; 13(3):221-227.

15. Abilés J, Lobo G, Pérez de la Cruz A, Rodríguez M, Aguayo E, et al. Valoración de La ingesta de nutrientes y energía en paciente crítico bajo terapia nutricional enteral. Nutr. Hosp. 2005; XX(2):110114.

16. Verrastro T. Hematologia e hemoterapia. São Paulo: Atheneu; 2005.

17. Blackburn GL, Thornton PA. Nutritional assessment of the hospitalized patients. Med. Clin. North Am. 1979; 63(5):11103-11115.

18. World Health Organization. Physical status: the use and interpretation of anthropometry. Geneve: WHO; 1995.

19. Waitzberg DL. Indicadores de qualidade em terapia nutricional. São Paulo: ILSI Brasil; 2008.

20. Henderson RC, Grossberg RI, Matuszewski J, Menon N, Johnson J, Kecskemethy HH, et al. Growth and nutritional status in residential center versus home-living children and adolescents with quadriplegic cerebral palsy. J. Pediatr. 2007; 151(2):161-166.

21. Tâmega IE, Barros Filho AA, Pinto EALC. Growth in children with encephalopathy, a longitudinal study from the 6th to the 24th month. Int. J. Nutr. Metab. 2011; 3(6):65-71.

22. Anderson L, Dobble MV, Turkki PR, et al. Nutrição. Rio de Janeiro: Guanabara Koogan; 1988.

23. Brasil. Ministério da Saúde. Diretrizes de atenção à pessoa com paralisia cerebral. Brasília: Ministério da Saúde; 2013.

24. Woodrow G. Body composition analysis techniques in the aged adult: indications and limitations. Curr. Opin. Clin. Nutr. Metab. Care. 2009; 12(1):8-14.

25. Heyward VH, Stolarczyk LM. Avaliação da composição corporal. São Paulo: Manole; 2000.

26. Rotta NT. Cerebral palsy, new therapeutic possibilities. J. Pediatr. 2002; 78(Supl. 1):S48-54.

27. McGowan JE, Fenton TR, Wade AW, Branton JL, Robertson M. An exploratory study of sodium, potassium, and fluid nutrition status of tube-fed nonambulatory children with severe cerebral palsy. Appl. Physiol. Nutr. Metab. 2012; 37(4):715-723.

28. Parrish CR. Serum proteins as markers of nutrition: what are we treating? Practical Gastroenterology 2006; 46-64. Nutrition Issues In Gastroenterology, Series, 43.

29. Obara H, Tomite Y, Doi M. Improvement in the nutritional status of very elderly stroke patients who received long-term complete tube feeding. e-SPEN, the European e-Journal of Clinical Nutrition and Metabolism 2010; 5(6):e272-e276. 
30. Bottoni A, Oliveira GPC, Ferrini MT, Waitzberg DL. Avaliação nutricional: exames laboratoriais. In: Waitzberg DL, editor. Nutrição oral, enteral e parenteral na prática clínica. 3a. ed. São Paulo: Atheneu, 2000. p. 279-294.

31. Gibson RS. Nutritional assessment: a laboratory manual. Oxford: Oxford University Press; 1993.

32. Monteiro CA, Szarfarc SC, Mondini L. Tendência secular da anemia na infância na cidade de São Paulo (1984-1996). Rev. Saúde Pública 2000; 34(Supl. 6):62-72.

33. Abreu J, Borno S, Montilla M, Dini E. Anemia y deficiência de vitamina a em ninõs evaluados em um centro de atención nutricional de Caracas. Arch. Latinoam. Nutr. 2005; 55(3):226-234.

34. Susin FP, Bortolini V, Sukiennik R, Mancopes R, Barbosa LR. Perfil de pacientes com paralisia cerebral em uso de gastrostomia e efeito nos cuidadores. Rev. CEFAC 2012; 14(5):933-942.

35. Silva AFF; Campos DJ; Souza MH; Schieferdecker MEM. Capacidade da terapia nutricional enteral em fornecer as necessidades calórico-proteicas de pacientes hospitalizados. Rev. Bras. Nutr. Clin. 2003; 18(3):113-8.

36. Martín FT, et al. Grupo de trabajo de gestión de senpe. Analysis of therelevance and feasibility of quality indicators in nutrition support. Nutri. Hosp. 2012; 27(1):198-204.

37. Jack L, Coyer F, Courtney M, Venkatesh B. Diarrhea risk factors in enterally tube fed critically ill patients: a retrospective audit. Intensive Crit. Care Nurs. 2010; 26(6):327-334.

38. Mostafa SM, Bhandari S, Ritchie G, Gratton N, Wenstone R. Constipation and its implications in the critically ill patient. Br. J. Anaesth. 2003; 91(6):815-819.

39. Tapia J, Murguia R, Garcia G, de los Monteros PE, Oñate E. Jejunostomy: techniques, indicators, and complications. World J. Surg. 1999; 23(6):596-602.

40. Malone AM, Seres DS, Lord L. Complications of enteral nutrition. In: Gottschlich MM, ed. The ASPEN nutrition support core curriculum: a case-based approach - the adult patient. Silver Spring, MD: American Society for Parenteral and Enteral Nutrition; 2007. p. 246-263.

41. Montejo JC. Enteral nutrition-related gastrointestinal complications in criticallyill patients: a multicenter study. The nutritional and metabolic working group of the spanish society of intensive care medicine and coronary units. Crit Care Med.1999; 27(8):1447-1453.

42. Doig GS, Heighes PT, Simpson F, Sweetman EA, Davies AR. Early enteral nutrition, provide within $24 \mathrm{~h}$ of injury or intensive care unit admission, significantly reduces mortality in critically ill patients: a meta-analysis of randomized controlled trials. Intensive Care Med. 2009; 35(12):2018-2027.

43. Bittencourt AF, Martins JR, Logullo L, Shiroma G, Horie L, Ortolani MC, et al. Constipation is more frequent than diarrhea in patients fed exclusively by enteral nutrition: results of an observational study. Nutr. Clin. Pract. 2012; 27(4):533-539.

Recebido: $14 / 1 / 2015$

Revisado: $16 / 1 / 2015$

Aprovado: 19/1/2015 